\title{
Anillo vascular completo por doble arco aórtico simétrico, una malformación cardiovascular infrecuente
}

\author{
Roberto González L. ${ }^{1,2}$, Camila Bustos C. ${ }^{1,2}$, Alberto Fuentes L. ${ }^{1,2}$, \\ Julio Yévenes S. ${ }^{1,2}$, Alejandra Riquelme U. ${ }^{1,2}$, Enrique Seguel S. ${ }^{1,2}$, \\ Aleck Stockins L. ${ }^{1,2}$, Andrés Jadue T. ${ }^{1,2}$, Emilio Alarcón C. ${ }^{1,2}$
}

${ }^{1}$ Hospital Clínico Regiona Dr. Guillermo Grant Benavente de Concepción. Concepción

Chile.

${ }^{2}$ Departamento de Cirugía Facultad de Medicina, Universidad de Concepción. Concepción, Chile.

Recibido el 25 de marzo de 2018 y aceptado para publicación el 22 de mayo de 2018.

Correspondencia a: Dr. Roberto Gonzalez L. rgonzalezlagos@udec.c
Complete vascular ring by double symmetrical aortic arch, an infrequent cardiovascular malformation

Introduction: The double aortic arch (DAA) is an uncommon cardiovascular malformation, characterized by the persistence of both aortic arches after birth. It can be presented with right dominant branch (70$73 \%$ ), left or symmetrical. It's anatomical shape produces compression of mediastinal structures such as the trachea and esophagus. Clinic case: Infant man 3 months old, with a history of stridor since birth. He was admitted to our center due to severe pneumonia with the need for prolonged mechanical ventilation. Evaluated by otorhinolaryngology, who performed airway revision observing stenosis in the last 5 tracheal rings with dynamic trachea collapse. Chest computed tomography showed complete DAA with emergence of supraaortic trunks of both arches symmetrically. Surgical treatment was decided as soon as possible. It was approached by left anterolateral thoracotomy, vascular control and left aortic arch section distal to subclavian emergency was performed, with aortic and left subclavian artery plasty, aortic arch was released to perform pexia and dissection of fibrotic tissue surrounding the trachea and esophagus. Patient evolved favorably, with resolution of respiratory symptoms and absence of stridor. Postoperative fiberoptic bronchoscopy did not observe tracheal compression. He was discharged on the 14th postoperative day. Currently at six months of follow-up, he is asymptomatic respiratory and cardiovascular. Discussion: AAD can affect $0.03 \%$ of the pediatric population. It is usually symptomatic with obstructive manifestations such as stridor or dysphagia due to compression of mediastinal structures, which requires high clinical suspicion for its imaging confirmation and subsequent surgical treatment.

Key words: aortopathies; aortic arch; thoracic surgery.

\section{Resumen}

Introducción: El doble arco aórtico (DAA) es una malformación cardiovascular infrecuente caracterizada por la persistencia de ambos arcos aórticos posterior al nacimiento. Puede presentarse con rama derecha dominante (70-73\%), izquierda o simétrica. Su forma anatómica produce compresión de estructuras mediastínicas como la tráquea y esófago. Caso clínico: Lactante menor hombre de 3 meses, con antecedente de estridor desde nacimiento. Ingresó a nuestro centro por neumonía grave con necesidad de ventilación mecánica prolongada. Evaluado por otorrinolaringología, se realizó revisión de vía aérea, observando estenosis en los últimos 5 anillos traqueales con colapso dinámico de la tráquea. La tomografía computada de tórax demostró DAA completo con emergencias de troncos supraaórticos de ambos arcos de forma simétrica. Se decidió tratamiento quirúrgico a la brevedad. Se abordó por toracotomía anterolateral izquierda, se realizó control vascular y sección del arco aórtico izquierdo distal a emergencia de subclavia, con plastía de aorta y arteria subclavia izquierda, liberado el arco aórtico se realizó pexia y disección de tejido fibrótico que rodeaba tráquea y esófago. Paciente evolucionó favorablemente, con resolución de cuadro respiratorio y ausencia estridor. Fibrobroncoscopía posoperatoria no observó compresión traqueal. El alta hospitalaria fue al $14^{\circ}$ día posoperatorio. Actualmente, a seis meses de seguimiento, se encuentra asintomático respiratorio y cardiovascular. Discusión: El DAA puede afectar al 0,03\% de la población pediátrica. Usualmente es sintomático con manifestaciones obstructivas como estridor o disfagia por compresión de estructuras mediastínicas, por lo que requiere alta sospecha clínica para su confirmación imagenológica y posterior tratamiento quirúrgico.

Palabras clave: aortopatías; arco aórtico; cirugía torácica. 


\section{Introducción}

Los anillos vasculares (AV) corresponden al conjunto de malformaciones congénitas del arco aórtico y/o de los grandes vasos torácicos, que rodean tanto al esófago como a la tráquea y que pueden dar sintomatología respiratoria y/o digestiva por comprensión extrínseca ${ }^{1-4}$.

Los AV corresponden cerca del $1 \%$ de todas las cardiopatías congénitas ${ }^{4}$. Anatómicamente se pueden dividir en: doble arco aórtico (DAA), arco derecho-ligamento ductal izquierdo, compresión de la arteria innominada y sling de la arteria pulmonar, siendo el primero el más frecuente ${ }^{4,5}$.

El DAA es una malformación cardiovascular infrecuente, que se define como la persistencia de ambos arcos aórticos, derecho e izquierdo, después del nacimiento, siendo dominante el arco derecho en el $70 \%-73 \%$ de los $\operatorname{casos}^{1,5,6}$.

El diagnóstico precoz del DAA es difícil, debido a que es una patología infrecuente y su sintomatología es poco específica, por lo que requiere alta sospecha clínica y estudios complementarios.

El tratamiento es quirúrgico. Si bien se recomienda el abordaje lateral por toracotomía, la esternotomía media es un adecuado abordaje quirúrgico en caso de concomitancia de alguna otra malformación cardiaca $^{5-8}$.

Se presenta un caso de un lactante menor, con diagnóstico de doble arco aórtico y sintomatología respiratoria obstructiva.

\section{Caso clínico}

Lactante menor hombre de 3 meses de edad, con antecedente de estridor, sintomático respiratorio desde el nacimiento y múltiples hospitalizaciones por infecciones respiratorias bajas.

Consultó, en hospital de origen, por deterioro del estado general, estridor y fiebre de 5 días de evolución sugerente de nuevo episodio de neumonía, sin síntomas digestivos, por lo que se decidió hospitalizar para tratamiento médico y estudio. Durante hospitalización evolucionó desfavorablemente con necesidad de hospitalización en unidad de paciente crítico y conexión a ventilación mecánica. Por evolución tórpida y necesidad de apoyo ventilatorio prolongado, fue derivado a nuestro centro para realización de traqueotomía.

Fue evaluado por equipo de otorrinolaringología por sospecha de estenosis subglótica. Se realizó revisión de vía aérea con laringotraqueoscopía con tubo rígido, observándose edema de bandas ventriculares, glotis y subglotis, estenosis subglótica grado
1 y estenosis en los últimos 5 anillos traqueales con colapso dinámico de la vía aérea. Se decidió completar estudio con tomografía computada de tórax, la que demostró un AV por DAA completo con emergencias simétricas de los troncos supraaórticos desde ambos arcos aórticos (Figuras 1A, 1B, 1C, 1D). Debido a la gravedad del cuadro clínico se decidió cirugía a la brevedad.

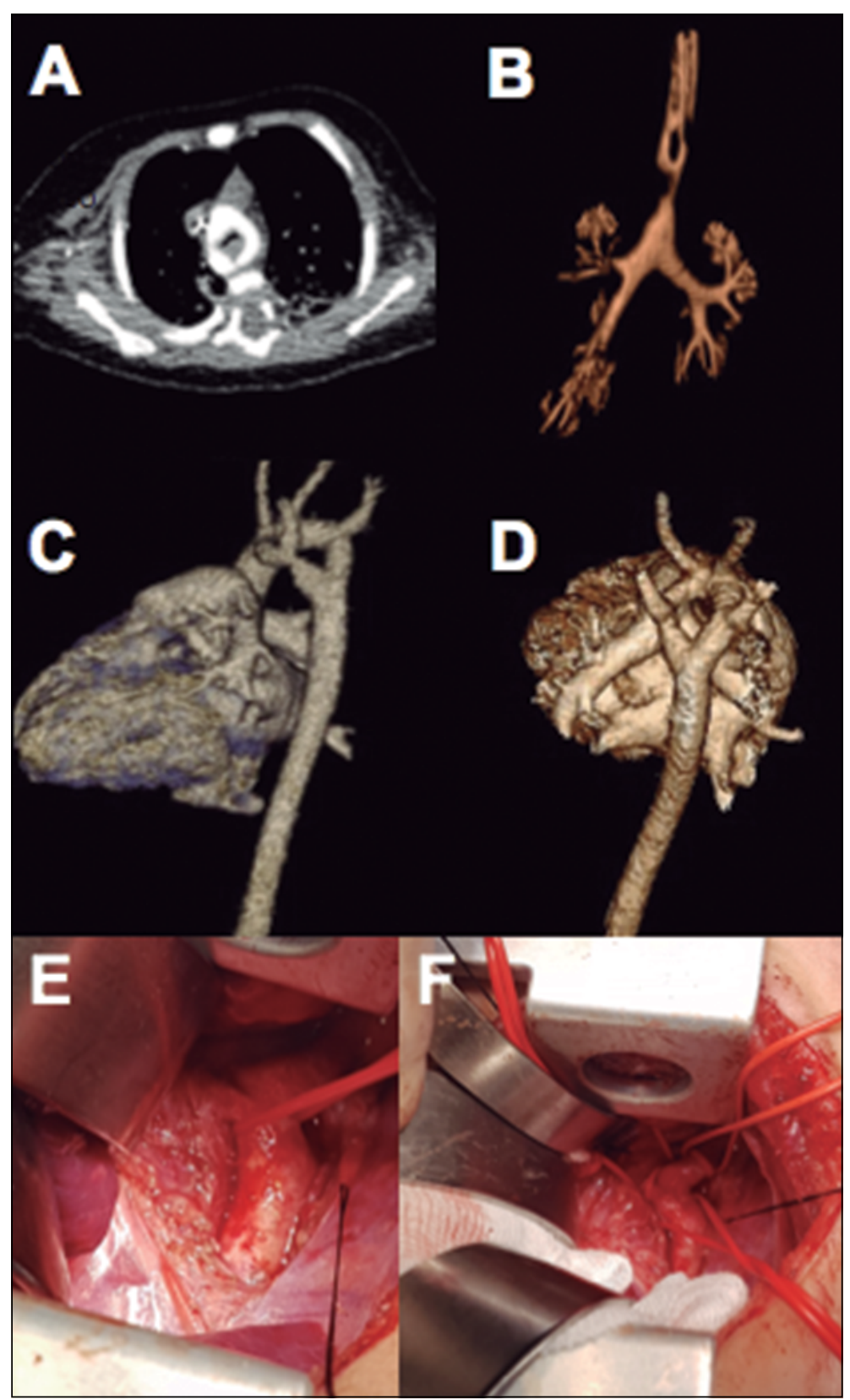

Figura 1. A, B: Tomografía computada de tórax evidenció DAA simétrico con comprensión extrínseca de esófago y tráquea. C, D: Reconstrucción 3D que evidenció DAA completo con emergencia de troncos supraaórticos desde ambos arcos aórticos. E, F: Imagen intraoperatoria donde se observó DAA completo. 
Por las características anatómicas se decidió abordaje por toracotomía izquierda. Se ingresó por $4^{\circ}$ espacio intercostal izquierdo, se observó DAA con arco izquierdo de aproximadamente $8 \mathrm{~mm} \mathrm{y}$ con arteria subclavia izquierda de $4 \mathrm{~mm}$ (Figuras $1 \mathrm{E}, 1 \mathrm{~F})$. Se inició disección de arteria subclavia izquierda hasta llegar a arco aórtico izquierdo, luego se liberó ampliamente arco izquierdo y se realizó control vascular y sección distal a emergencia de arteria subclavia con plastía de la aorta y arteria subclavia izquierda, posteriormente, se liberó el arco aórtico y ligamento arterioso, completándose pexia y disección de tejido fibrótico que rodeaba tráquea y esófago, logrando la liberación de las estructuras mediastínicas.

Evolucionó favorablemente en el posoperatorio, con resolución completa de sintomatología respiratoria, ausencia de estridor y retiro de ventilación mecánica aproximadamente a las 48 horas. Fibrobroncoscopía de control no observó compresión traqueal. Por buena evolución clínica, el alta hospitalaria fue al $14^{\circ}$ día posoperatorio.

Actualmente, a 6 meses de seguimiento, se encuentra en buenas condiciones clínicas sin estridor, con adecuado desarrollo pondoestatural.

\section{Discusión}

Las malformaciones del arco aórtico corresponden a menos del 1\% de las alteraciones congénitas cardiovasculares ${ }^{3}$. Éstos suelen ser asintomáticos, salvo que compriman estructuras mediastínicas como el esófago y la tráquea. El DAA es el AV más frecuente, afectando al $0,03 \%$ de la población pediátrica y correspondiendo al 33\%-73\% de todos los $\mathrm{AV}^{3-6}$.

Las múltiples malformaciones del arco aórtico se pueden producir por una serie de alteraciones en el desarrollo y/o regresión de uno o varios componentes de la vasculogénesis durante la embriogénesis. En el caso del DAA, este se forma por la persistencia del cuarto arco y los vasos dorsales, formando un $\mathrm{AV}$ completo rodeando las estructuras mediastínicas, los cuales se unen para originar la aorta descendente, que se localiza generalmente a la izquierda, aunque también se puede observar a la derecha o en línea media del mediastino posterior $^{3-5}$. El DAA se puede presentar con rama derecha dominante (70\%-73\%), rama izquierda dominante (18\%-25\%), simétrico (9\%-5\%) y raramente con un arco hipoplásico ${ }^{1,4-6}$. En nuestro caso, se presentó un DAA simétrico con aorta descendente a la izquierda.

El DAA, por su forma anatómica, es general- mente sintomático. Sin embargo, cada vez se logran más diagnósticos de AV y DAA con ecografías prenatales, realizándose tratamientos quirúrgicos en pacientes asintomáticos ${ }^{8,9}$. El DAA se presenta clínicamente, dependiendo de su tamaño y extensión, en el 91\% con síntomas respiratorios obstructivos como estridor, infecciones respiratorias a repetición, broncoespasmos y/o en el $40 \%$ con síntomas digestivos como disfagia, dificultad en la alimentación y alteración del desarrollo pondoestatural. Además, la comprensión extrínseca de la vía aérea puede alterar el desarrollo y crecimiento normal del árbol bronquial ${ }^{5,6}$. En nuestro caso, al igual que en otros casos publicados, los síntomas clásicos fueron el estridor y las infecciones respiratorias a repetición. No se encontró antecedente de ecografía prenatal con diagnóstico de DAA.

Si bien el DAA presenta síntomas más tempranamente que otros $\mathrm{AV}$, su diagnóstico requiere una alta sospecha clínica, ya que su sintomatología, poco específica, obliga a descartar otras patologías neonatales de mayor frecuencia. Debido a que la mayoría de los DAA se diagnostican en la etapa posnatal, el estudio se inicia, de acuerdo a la sintomatología predominante, con una fibrobroncoscopía o con estudio contrastado de esófago, sin embargo, la tomografía computada, principalmente la angiotomografía computada (AngioTC) es el examen de elección para un diagnóstico correcto y una planificación quirúrgica adecuada al observar la correcta relación del DAA con la tráquea, esófago y otras estructuras mediastínicas ${ }^{1,5,6}$. La ecocardiografía se utiliza principalmente para descartar la presencia de alguna anomalía congénita intracardiaca asociada, la cual se observa en el $7 \%$ al $17 \%$ de los $\operatorname{casos}^{5,6,10,11}$. La comunicación interventricular corresponde a la malformación intracardiaca más frecuentemente observada; también se describe asociación con comunicación interauricular, ductus arterioso persistente, tetralogía de Fallot, etc ${ }^{10,11}$.

El tratamiento quirúrgico oportuno en pacientes con compresión extrínseca de las estructuras mediastínicias es imperioso para prevenir alteraciones en el desarrollo de la vía aérea y complicaciones a largo plazo $^{5,7}$. El abordaje quirúrgico de elección es la toracotomía lateral izquierda (78\%), reservándose la toracotomía lateral derecha en caso de arco izquierdo dominante o aorta descendente derecha y la esternotomía media principalmente frente a una concomitancia con otra malformación intracardia$\mathrm{ca}^{6-8}$. La cirugía logra solucionar la sintomatología aproximadamente en el $70 \%-92 \%$ de los casos $^{5}$. Algunos autores recomiendan la realización de una revisión de vía aérea posoperatoria, ya que se 
describen casos de falta de reexpansión de la vía aérea pese a liberación del anillo vascular, siendo la principal causa de reintervención quirúrgica ${ }^{5-7}$. En nuestro caso se realizó tratamiento quirúrgico rápidamente una vez hecho el diagnóstico, abordándose por toracotomía lateral izquierda sin presencia de nueva compresión traqueal al control con fibrobroncoscopía.

En conclusión, DAA es una anomalía congénita cardiovascular infrecuente, que se presenta clínicamente con síntomas respiratorios y digestivos por comprensión extrínseca tanto de la tráquea como del esófago. Se necesita una alta sospecha clínica, ya que obliga a descartar otras patologías de mayor frecuencia. El AngioTC es el estudio diagnóstico de elección. Requiere un tratamiento quirúrgico precoz para mejorar la sintomatología, prevenir estenosis residual y alteración en el desarrollo y crecimiento de la vía aérea.

\section{Responsabilidades éticas}

Protección de personas y animales. Los autores declaran que para esta investigación no se han realizado experimentos en seres humanos ni en animales.

Confidencialidad de los datos. Los autores declaran que en este artículo no aparecen datos de pacientes.

Conflicto de intereses: No hay.

\section{Referencias}

1. Das S, Nair V, Airan B. Double aortic arch as a source of airway obstruction in a child. Ann Card Anaesth. 2015;18:111-2.

2. Becit N, Erkut B, Karaca Y. Vascular ring, tracheoesophageal compression associated with symmetrical double aortic arch. Tex Heart Inst J. 2008;35:209-10.

3. Vera E, Martínez M, Marín A, Galdeano JM, García M. Anillos vasculares completos. An Pediatr. (Barc) 2008;69:525.

4. Rioseco P, Ahumada A, Vásquez D. Anillos vasculares como diagnóstico diferencial del asma. Rev Chil Enferm
Respir. 2012;28:51-7.

5. Sánchez R, Rey J, Polo L, Aroca A, González A, Pérez R, et al. Anillos vasculares y compresión traqueoesofágica: 15 años de experiencia quirúrgica. Cir Cardiovasc. 2016;23:11924.

6. Kaldararova M, Simkova I, Varga I, Tittel P, Kardos M, Ondriska M, et al. Double aortic arch anomalies in children: A systematic 20-year single center study. Clin Anat. 2017;30:929-39.

7. Richardson J, Doty D, Rossi N, Ehrenhaft J. Operation for Aortic Arch Anomalies. Annals Thorac Surg. 1981;31:426-32.

8. Dan Z, Qiangfu H, Liang T, Changyu L,
Linnan D, Laichun S. Repair of double aortic arch in patients with concomitant cardiac anomalies. J Card Surg. 2016;31:635-8

9. Gou Z, He Y, Zhang Y, Gu X. Prenatal diagnosis of fetal double aortic arch: report of case. Int J Clin Exp Pathol. 2015;8:13565-7.

10. Rock A, Eltayeb O, Camarda J, Gotteiner N. Prenatal diagnosis of the rare association of common arterial trunk and double aortic arch. Clin Case Rep. 2016;3:668-70

11. Cui W, Patel D, Husayni T, Roberson D. Double aortic arch and D-transposition of the great arteries. Echocardiography 2008;25:91-5. 